\title{
GT-Bidding: Group Trust Model of P2P Network Based on Bidding
}

\author{
Lin Zhang $\mathbb{D}^{1,2}$ Xinyan Wei, ${ }^{1}$ Yanwen Huang, ${ }^{1}$ Haiping Huang $\mathbb{D}^{1,2}$ Xiong Fu ${ }^{1},{ }^{1,2}$ \\ and Ruchuan Wang ${ }^{1,2}$ \\ ${ }^{1}$ School of Computer, Nanjing University of Posts and Telecommunications, Nanjing 210003, China \\ ${ }^{2}$ Jiangsu High Technology Research Key Laboratory for Wireless Sensor Networks, Nanjing 210003, China
}

Correspondence should be addressed to Lin Zhang; zhangl@njupt.edu.cn

Received 19 July 2020; Revised 22 March 2021; Accepted 17 June 2021; Published 9 July 2021

Academic Editor: Shehzad Ashraf Chaudhry

Copyright (c) 2021 Lin Zhang et al. This is an open access article distributed under the Creative Commons Attribution License, which permits unrestricted use, distribution, and reproduction in any medium, provided the original work is properly cited.

Due to the lack of trusted third parties as guarantees in peer-to-peer (P2P) networks, how to ensure trusted transactions between peers has become a research hotspot. However, the open and distributed characteristics of P2P networks have brought challenges to network security, and there are problems such as node fraud and unavailability of services in the network. To solve the problem of how to select trusted transaction peers in P2P groups, a new trust model, GT-Bidding, is proposed in this paper. This model follows the bidding process of human society. First, each service peer applies for a group of guarantee peers and carries out credit mortgages for this service. Second, based on the entropy and TOPSIS method (Technology for Order Preference by Similarity to an Ideal Solution) approaching the ideal solution, a set of ideal trading sequences is selected. Then, the transaction impact function is used to assign weights to the selected guarantee peers and service nodes, respectively; thus, the comprehensive trust of each service node can be calculated. Finally, the service peer is verified using feedback based on the specific confidence level, which encourages the reputation of the service and its guarantee peers to update. Experiments show that GT-Bidding improves the successful transaction rate and resists complex attacks.

\section{Introduction}

With the continuous development of network technology, P2P networks have been widely used because of their anonymity and openness, such as in file sharing and instant messaging. Nevertheless, these characteristics also bring new challenges to $\mathrm{P} 2 \mathrm{P}$ networks. The random spread of illegal messages by malicious peers will have negative impacts on the data security [1] and user experiences [2]. Moreover, the dynamic and distributed nature of the P2P network makes it difficult for peers to conduct long-term and stable transactions [3].

In the traditional $\mathrm{C} / \mathrm{S}$ architecture, one or more fixed machines are often used as servers to provide resources for all clients. Although this structure can reduce the communication load of the system, it often leads to single point failures. In the P2P network structure, each peer is not only a resource provider but also a resource acquirer. Due to the lack of centralized management of the central server, the problem of trust in the $\mathrm{P} 2 \mathrm{P}$ network urgently needs to be solved. The needed resources may exist between multiple peers $\left\{P_{1}, P_{2}, \ldots, P_{n}\right\}$. How to select the service peer with the highest comprehensive trust is the key research topic of this paper.

In recent years, cloud computing [4] has developed rapidly due to its excellent model. It allows on-demand access to adaptive resources and functions, making P2P technology widely used in traditional networks, but it puts forward new requirements for P2P security. He et al. [5] designed an efficient and fine-grained data access control mechanism (ACPC) for P2P cloud storage and an encryption scheme $(\mathrm{ABE})$ based on ciphertext policy attributes and proxy re-encryption, which could implement access policies based on user attributes and integrate them in the ACPC P2P reputation system. Patel and Sethi [6] proposed a hybrid-based authentication scheme called "Enhanced Virtual Password Authentication (EVPA)," which aimed to implement a virtual password mechanism to resist shoulder surfing attacks, using the string part of the password and mathematical functions value to protect the user password. 
Mechanisms such as access control and encryption can control the access to the secure node before the guarantee. During the guarantee process, encryption can be used to ensure the reliability of the transaction, thereby improving the security performance.

In order to solve the above problems better, this paper proposes a new trust model GT-Bidding and designs the following improvements:

(1) The entire transaction process is planned for bidding. Each service node needs to apply for a group of guarantee nodes to mortgage the reputation of the service to gain the opportunity to obtain the service. This not only guarantees the reliability of the service node but also dynamically calculates the trust value through the trust update of the guarantee node.

(2) Information entropy is used as the weight of the evaluation indicator to avoid the arbitrariness and subjectivity of the weight setting in the traditional trust mechanism. According to the TOPSIS selection method, the service nodes are sorted in order to obtain the ideal transaction node sequence. The comprehensive trust calculation is performed to obtain the final transaction node.

(3) To avoid cooperative cheating and other attacks, the GT-Bidding model implements feedback verification. After verification, the reputations of the service peer and guarantee peers are updated according to the feedback information.

(4) In terms of computational complexity, the super node undertakes most of the computational functions, such as guaranteeing the trust of the node and verifying and updating the trust value of the service node, thereby reducing the node overhead and reducing the computational complexity.

The organization structure of this paper is as follows.

Section 2 analyses the existing trust models at home and abroad. Section 3 describes the GT-Bidding model in detail and gives the relevant definitions. Section 4 discusses the specific algorithm flow of a service peer applying for guarantee peers. Section 5 proposes a preference selection method based on entropy to rank the service peers and obtain the ideal transaction sequence. Section 6 combines the global trust with the guaranteed trust to calculate the comprehensive trust. Section 7 discusses how to verify the feedback of the requesting peer and how to update the reputations of the service peer and the guarantee peers. Section 8 is the simulation experiment and result analysis. Section 9 is the summary of this paper and the planned future work.

\section{Related Works}

The research focus at home and abroad has become how to establish a reliable and secure trust model to supervise and restrict the behavior of peers in $\mathrm{P} 2 \mathrm{P}$ networks, which will allow peers to make up for the shortcomings of the lack of a central server and a trusted third party by evaluating each other's transactions and giving feedback as well as letting users choose the most trusted peers for transactions.

Kamvar and Schlosser [7] proposed a classical global trust model based on recommendations. The concept of a prior trust peers is proposed. It is assumed that all peers have a priori trust in the initial stage of the network's establishment. Each peer $P_{i}$ has a unique global trust, and any peer requesting $P_{i}^{\prime}$ s service has local trust. The trust model calculates the global trust of $P_{i}$ by aggregating the local trust of all peers, but it does not consider the dynamic reliability of the peers in the network. As a result, it has the disadvantages of poor scalability and high computational complexity. The model is not suitable for large-scale P2P networks. Tian et al. [8] proposed a SuperTrust model based on aggregated super peers in which peers are clustered by interest sets, and the trust relationships were divided into three categories: trust between super peers, trust between super peers and ordinary peers, and trust between ordinary peers. They also gave specific trust measurement algorithms. However, due to the lack of consideration of uncertainties in the recommendation information itself, it is difficult to effectively judge the quality of service.

Shen et al. [9] proposed a hybrid trust model based on preference similarity, which integrates global trust and local trust. The sparse trust matrix is optimized using the similar random walk strategy. Wu [10] proposed an SGTM model based on the enhanced stable group, presented the construction algorithm of the stable group, effectively partitioned the MP2P network, and maintained relatively stable trust relationships and topology among the same group peers; however, this study lacked the filter processing of feedback information and could not discard false evaluations and avoid malicious aggressive feedback. Wang and Ju [11] proposed the MeTrust model, which divides all the evidence affecting trust into three different layers, the graphics layer, the peer layer, and the path layer, and evaluates the trust from the perspective of personalization. Qureshi et al. [12] proposed the $\mathrm{M}$-Trust model, gave the aggregation algorithm of distributed trust, and calculated the trust using the recommendations of direct interaction peers and other peers. However, it is not easy to determine whether the recommender is trustworthy in the dynamic P2P network environment, and the trust threshold limit needs to be adjusted according to the specific situation of the network. Samah et al. [13] proposed an extended Advogato based on the cluster trust evaluation method, which is different from the traditional Advogato method. It combines the strength of social relations to find the reliable user groups that are associated with each individual user. By extending the trust measure, it designs a diffusion mechanism to ensure that the ability of each peer can effectively follow the social relationship connection chain. It extends to continuous peers, identifies local users by maximizing the flow of the design capability, and divides the trust level according to the degree of trust. This forms a reliable and orderly set of trust users, prevents malicious users from accessing personal networks, and protects the information content of reliable users.

Das and Islam [14] proposed a model that can effectively calculate the dynamic trust of peers, even if there are highly oscillating malicious behaviors in the network. To prevent 
malicious peers from strategic oscillation and constantly improve or reduce their reputation values, the SecuredTrust model evaluates the behavior of peers by investigating the historical trust of peers and uses the cumulative frequency of the trust fluctuation to measure the deviation of peer behavior. Accordingly, if the behavior of peers oscillates, the trust of peers will be reduced, and a load balancing algorithm should be adopted. This algorithm assigns service requests to all capable peers, which solves the problem of complex and overloaded communication between peers with high trust, but it has high computational complexity. Hu et al. [15] proposed a social P2P network in which each peer can find partners through a social tracker according to the common interests of the peers and maintain long-term stable social communications with them. It also proposes a distributed trust mechanism. The trust between two friends can reflect the level of cooperation between them. They communicate with each other. Although social trust can withstand more forms of attacks, it does not take malicious social trackers into account. Meng [16] proposed a super-peer perception model based on a two-level P2P network, which allows only peers with similar trust values to trade.

The PROVEST model [3] is a trust model based on provenance, which can dynamically evaluate the trust of peers according to the changes of the environment and peer conditions [17]. At present, trust models based on fuzzy theory [18] and cloud theory [19] are also studied, which combine uncertain descriptions between peers with trust descriptions. However, the computational complexity of such algorithms is high, and the resistance to multiple malicious combination attacks [20] is still insufficient.

Ma et al. [21] proposed an approach called Distributed Consensus-Based Trust Model (DCONST), which can evaluate the trustworthiness of IoT nodes by sharing certain information called cognition under multiple-mix-attack model where attackers can launch several attacks simultaneously. Meng et al. [22] applied blockchains for enhancing the effectiveness of Bayesian inference-based trust management to detect malicious nodes in MSNs, which can help improve the detection efficiency of malicious nodes. Narang and Kar [23] proposed a hybrid trust management framework that makes use of Probabilistic Neighborhood Overlap (P-NO), which shows the effectiveness of the proposed framework in handling different attack scenarios.

In summary, the existing trust models have achieved some improvements in resisting the malicious attacks of peers [24], but when there are multiple service peers, how to avoid malicious behaviors such as cooperative cheating among peers [25], reduce the time complexity, rationally reward and punish peers [26], and mobilize the enthusiasm of peers to provide services remains unsolved. The bidding model proposed in this article can effectively solve the above problems. Experiment 1 shows the reduction of computational complexity. Experiments 2 to 4 show the ability to resist three types of malicious node attacks, such as complete malicious peer, defame peer, and strategic malicious peer. Experiments 5 to 6 show the ability to resist cooperative cheating. The reputation updating mechanism can guarantee the reward and punishment of nodes.

\section{Trust-Based P2P Group Bidding Model}

To conveniently understand the specific flow of the GTBidding algorithm, this paper first introduces several important definitions.

Definition 1 (service peer (SP)). The service peer is also known as the transaction peer or target peer and refers to the peer providing services in the transaction process. A service peer has a service reputation. The service reputation is the reliability of the services that are provided by service peers.

Definition 2 (request peer (RP)). The request peer is the peer requesting service in the transaction process. After the transaction is completed, the request peer needs to send the feedback from the service peer and the guarantee peer to their respective super peers.

Definition 3 (super peer (SuP)). Super peers are responsible for managing the service reputation, guaranteed reputation, and transaction records of all peers in their group.

Definition 4 (guarantee peer). The guarantee peer is the peer that provides credit guarantees for service peers in each transaction. The guaranteed peer has a guaranteed reputation. The guarantee reputation represents the credibility of the guarantee that is provided by the guarantee peer. When the transaction succeeds, the guarantee peer will be rewarded with reputation, and a newly joined peer can accumulate reputation through the guarantee process.

Definition 5 (direct trust $\left(\mathrm{DT}_{i j}\right)$ ). Direct trust represents the trust evaluation of service peer ${ }_{i}$ by request peer ${ }_{j}$ based on its historical transaction records (number of successful transactions/total transactions).

Definition 6. Service bidding messages $(\mathrm{SBM})=\left(R_{i} \mid G_{i}\right.$, Reward, Resource), where $R_{i}$ is the request peer, $G_{i}$ is the i.d. of the group where the request peer is located, Reward is the reputation reward that guarantee peers and the service peer can obtain after the service is completed, and Resource is the resource content of the request.

Definition 7. Service application messages $(\mathrm{SAM})=\left(S_{j} \mid G_{j}\right.$, GESET, ROT), where $S_{j}$ is the service peer, $G_{j}$ is the group i.d. where the service peer is located, and GESET is the guarantee peer sequence $\left\{g_{1}, g_{2}, \ldots, g_{k}\right\}$ of the service peer. ROT is the remaining online time of the peer. To avoid the sudden disconnection of the peer that is providing resources when the file resources are downloaded to approximately $90 \%$, the peer with a long remaining online time should be selected. Since this article only discusses the process of requesting service once, all service applications respond only to the unique SBM.

The GT-Bidding model is shown in Figure 1. The model is divided into six parts. Each group consists of several common peers and one super peer. The first part is the request peer to issue bidding information. The second part is the service peer that applies for a group of guarantee peers 


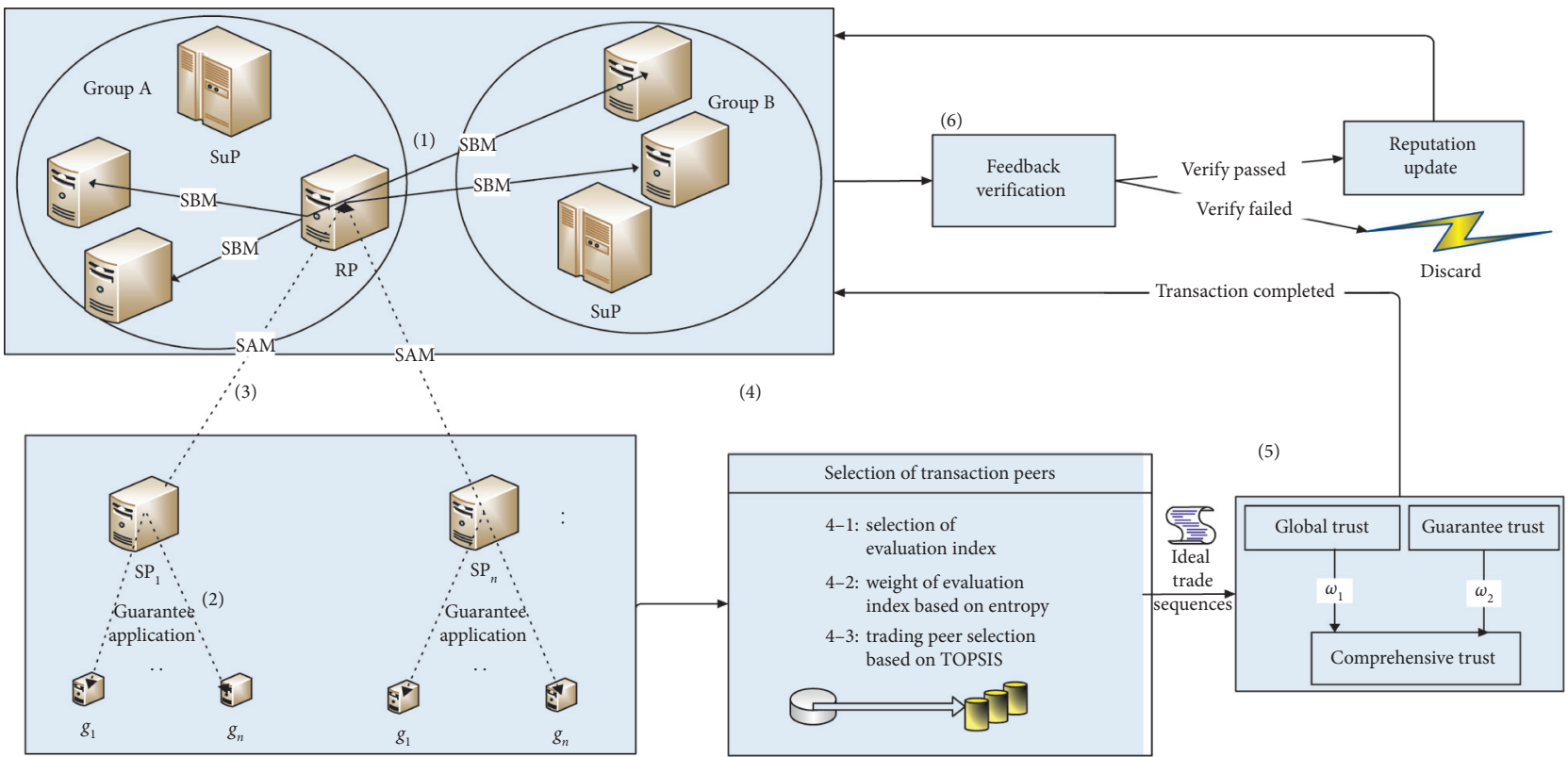

Figure 1: GT-bidding model.

from the whole network. This paper only considers that one guarantee peer can only guarantee one request peer at a time. The third part is the request peers returning the SBM to the service peer. The fourth part is the selection of the ideal service peer, the fifth part is the comprehensive trust calculation, and the sixth part is the feedback verification of the request peer. If the verification fails, the feedback information is discarded. Otherwise, the reputation value of the service peer and the guarantee peers will be updated.

The specific algorithm steps are described as follows:

Step 1 . The request peer $R_{i}$ publishes the service bidding document SBM to the whole network, and the service peer $S_{j}$ responds to the SBM.

Step 2. Service peer $S_{j}$ applies for a guarantee peer to the whole network, and the sequence of guaranteed peers $\left\{g_{1}, g_{2}, \ldots, g_{k}\right\}$ is obtained.

Step 3. The service peer $S_{j}$ returns its service application SAM.

Step 4 . The sequence of all service peers $\left\{S_{1}, S_{2}, \ldots, S_{n}\right\}$ participating in the bidding is obtained. The service peers are sorted using the TOPSIS method, and a new service sequence $\left\{S_{1}^{\prime}, S_{2}^{\prime}, \ldots, S_{n}^{\prime}\right\}$ after the resource ranking is obtained.

Step 5. The global trust and guarantee trust between the bidding service peer and the request peer are added using different weights to obtain the comprehensive trust of the service peer, and the peer with the highest comprehensive trust is selected as the final transaction peer.

Step 6. If the transaction is completed, the request peer gives feedback to the service, and the super peer in the group where the service peer is located verifies the feedback. If the FM is judged to be validated within the confidence interval, the service peer and its guaranteed peers need to be updated.

The description of the GT-Bidding algorithm based on the bidding process in a P2P network is shown in Algorithm 1.

\section{Guarantee Process}

Definition 8 (remaining guarantee reputation (RGR)). The residual guaranteed reputation is the valid reputation that the current guaranteed peer can use for guaranteeing.

$$
\mathrm{RGR}_{g}=\mathrm{RGR}_{g}^{\mathrm{pre}}-\sum_{i=1}^{m} \mathrm{RM}_{g}^{i},
$$

where $\mathrm{RGR}_{g}^{\text {pre }}$ is the current guarantee reputation of guarantee peer $g, \mathrm{~m}$ is the number of guarantee peers, and $\mathrm{RM}_{g}^{i}$ is the reputation that is guaranteed by peer $g$ in the ith service. $\mathrm{RGR}_{g}^{\text {pre }}$ and $\mathrm{RM}_{g}^{i}$ are obtained from the super peer of the group where peer $g$ is located.

If the service peer wants to provide service, at least one guarantee peer is needed to guarantee the service. To improve the credibility of the transaction, the super peer needs to judge whether the guarantee peer is qualified to guarantee and only through the audit of the guarantee will it take effect. The guarantee process is divided into the following six parts, which is shown in Figure 2:

(1) Application by service peer: Service peer $S_{j}$ issues the application $\left(S_{j}\left|G_{j}, R_{i}\right| G_{i}\right)$ to the super peer of each group, where $G_{j}$ is the group i.d. of service peer $S_{j}$ and $G_{i}$ is the group i.d. of request peer $R_{i}$.

(2) Review service peers: When each group of super peers receives the application of the service peer $S_{j}$, there are two cases as follows: 


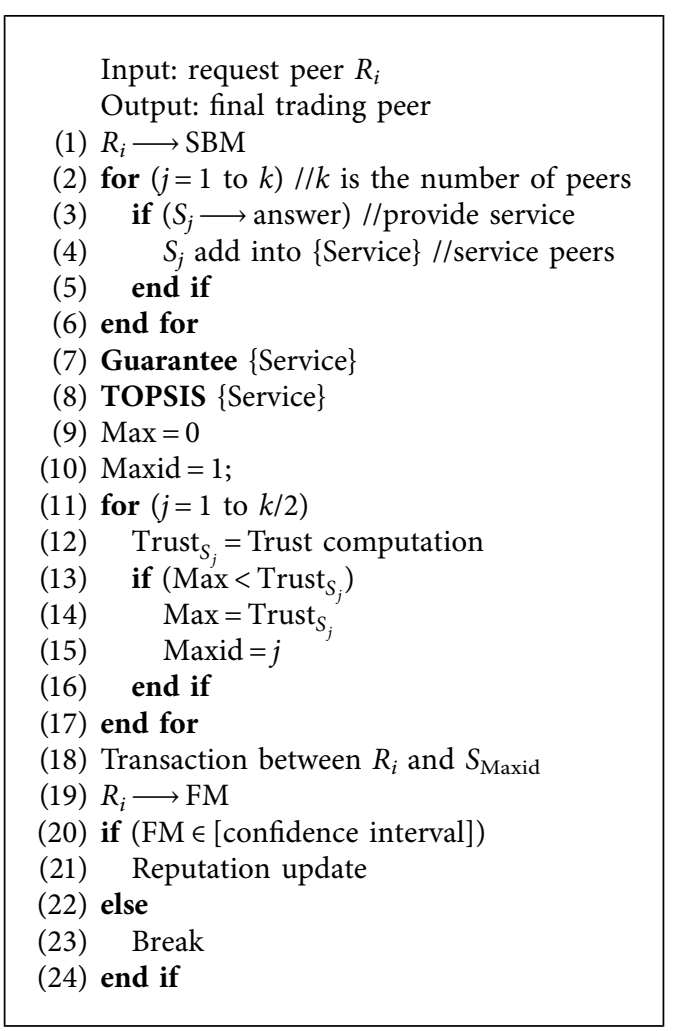

Algorithm 1: GT-bidding algorithm.

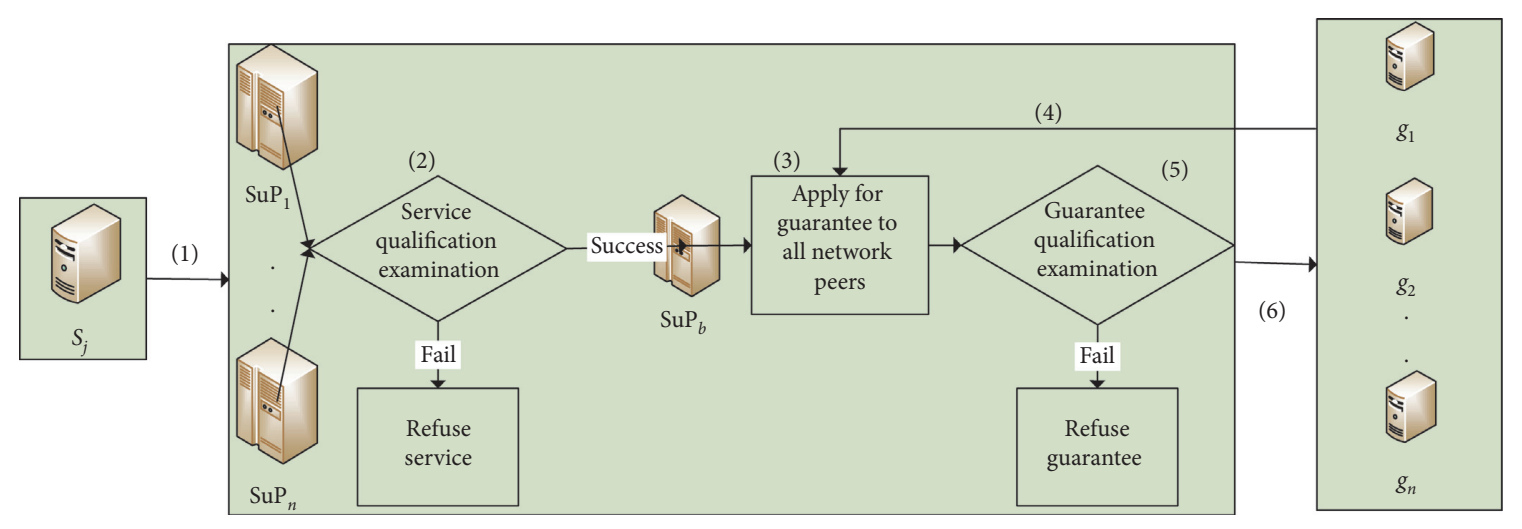

Figure 2: Guarantee process.

(i) If $G_{i}=G_{j}=a$, then the service peer $S_{j}$ and the request peer $R_{i}$ are in group $a$. Then, the local trust $\mathrm{LC}_{i}(j)$ of $R_{i}$ to $S_{j}$ is as follows:

$$
\operatorname{LC}_{i}(j)= \begin{cases}\frac{N_{i j}^{S}-N_{i j}^{F}}{N_{i j}}, & N_{i j}=N_{i j}^{S}+N_{i j}^{F} \text { and } N_{i j} \neq 0, \\ 0, & \text { other, }\end{cases}
$$

where $N_{i j}^{S}$ is the number of successful transactions and $N_{i j}^{F}$ is the number of failed transactions. If there is no transaction record, the local trust is 0 .
Then, the global trust of $R_{i}$ to $S_{j} D_{s}^{t_{k}}(i, j)$ is calculated as follows:

$$
D_{s}^{t_{k}}(i, j)=\sum_{t_{k}=1}^{n} \frac{d\left(t_{k}\right) * \mathrm{LC}_{i}(j)}{\sum_{t_{k}=1}^{n} d\left(t_{k}\right)}
$$

where $d\left(t_{k}\right)$ is a time attenuation function. It is defined as follows:

$$
d\left(t_{k}\right)=e^{-L\left(t_{k}\right)},
$$

where $t_{k}$ is the time point for the kth service and $L\left(t_{k}\right)$ is the distance between each transaction and the current time $t_{0}$. In this paper, the 
reference time is 90 days or three months, and there are 30 days per month.

Therefore, $L\left(t_{k}\right)=\operatorname{round}\left(\left(t_{k}-t_{0}\right) / 30\right)$ is an integral function, the minimum distance is $0 / 30$, and the maximum distance is $90 / 30$. There are more than 90 days according to 90 days to calculate attenuation.

(ii) $G_{i}=a, G_{j}=b$, and $a \neq b$. At this time, the request peer $R_{i}$ and the service peer $S_{j}$ are in group $a$ and group $b$, respectively. Because of the high similarity of interest sets between the same group and the rich interaction between the peers, the trust measures between the peers in the same group can be relatively accurate based on a large number of transaction records. Meanwhile, the interest sets of the different group peers are quite different and the transaction records are relatively sparse, which makes it necessary to share their interest sets between group $a$ and group $b$. The trading experience of other peers determines the measure of trust. The trust of group $a$ for group $b$ is as follows:

$\mathrm{TR}_{a, b}= \begin{cases}N_{a, b}^{S}-\frac{N_{a, b}^{F}}{N_{a, b}}, & N_{a, b}=N_{a, b}^{S}+N_{a, b}^{F} \neq 0, \\ 0, & N_{a, b}=N_{a, b}^{S}+N_{a, b}^{F}=0,\end{cases}$

where $N_{a, b}^{S}$ is the number of successful transactions between group $a$ and group $b$ and $N_{a, b}^{F}$ is the number of failed transactions between group $a$ and group $b$. Formula (5) is group $a$ 's trust in service peer $S_{j}$ :

$$
\mathrm{TR}_{a, S_{j}}=\frac{\sum_{p_{i}^{a} \in I\left(s_{j}\right)}\left(N_{p_{i}^{a}, p_{j}}^{S}-N_{p_{i}^{a}, S_{j}}^{F}\right)}{\sum_{p_{i}^{a} \in I}\left(s_{j}\right)^{N} N_{p_{i}^{a}, S_{j}}},
$$

where $I\left(S_{j}\right)$ is the set of peers that interact with $S_{j}$ in group a. $R_{i}$ 's global trust in $S_{j} D_{s}^{t_{k}}(i, j)$ is calculated as follows:

$$
D_{s}^{t_{k}}(i, j)=\frac{\mathrm{TR}_{a, b} * \mathrm{TR}_{a, S_{j}} * \sum_{t_{k}=1}^{n} d\left(t_{k}\right) * \mathrm{DT}_{j i}}{\sum_{t_{k}=1}^{n} d\left(t_{k}\right)_{j i}}
$$

(3) Waiting for guarantee peer response: When the service peer $S_{j}$ passes the audit, the super peer $\mathrm{SuP}_{b}$ applies for a guarantee to the whole network.

(4) Guarantee peer issues notification: Ordinary peer $g_{k}$ receives a guarantee request from service peer $S_{j}$ and responds with Response $\left(g_{k} \mid G_{k}, S_{j}\right)$.

(5) Verify whether the guarantee peer is qualified for the guarantee.

(6) Provide guarantee: Peer $g_{k}$ sends Notify $\left(S_{j} \mid G_{j}\right.$, $g_{k} \mid G_{k}$ ) to service peer $S_{j}$, where $G_{k}$ is the group i.d. of guarantee peer $g_{k}$.
By calculating the trust value of the guarantee node sequence, we can obtain a set of reliable guarantee sequences. The specific guarantee algorithm is described as Algorithm 2.

\section{Entropy-Based TOPSIS Service Peer Selection Method}

To objectively give the weight of the service evaluation index, this paper introduces the TOPSIS selection method based on entropy and uses the TOPSIS selection method to obtain the optimal solution. In addition, to avoid the subjectivity of data, this paper uses the entropy method to determine the weight of each index. By sorting the service peers, the ideal sequence of transaction peers is obtained, which reduces the computational overhead.

In 1981, Yoon and Hwang first proposed the TOPSIS (Technology for Order Preference by Similarity to an Ideal Solution) method, which is also known as ideal point method and is based on approaching the ideal solution. This method ranks the proximity distance between the evaluation object and the idealized object, and its basic idea is as follows. The TOPSIS method detects the relationship between the evaluation object and the worst solution and the optimal solution. The distance ranking is the best if the evaluation object is nearest to the optimal solution and farthest away from the worst solution; otherwise, it is the worst. Each index value of the optimal solution reaches the optimal value of each evaluation index, and each index value of the worst solution reaches the worst value of each evaluation index.

(1) Construct the decision matrix: $X=\left\{x_{1}, x_{2}, \ldots, x_{m}\right\}$ represents $m$ service peers. According to the trust relationship between the peers in the P2P network, this paper selects five evaluation indicators, which are the remaining online time, the successful transaction rate, the comprehensive guarantee reputation, the service content, and the response time. The five evaluation indexes are represented by $Y=$ $\left\{y_{1}, y_{2}, y_{3}, y_{4}, y_{5}\right\}$ (Table 1 ).

(2) Normalization is as follows:

$$
Z_{i j}=\frac{y_{i j}}{\sqrt{\sum_{i=1}^{m} y_{i j}^{2}}}, \quad i=1, \ldots, m, j=1, \ldots, 5 .
$$

(3) Solve the index weight using the entropy method: The weight of each index is usually determined using the ARP (analytic hierarchy process), which is a subjective method to determine the weight based on the idea of expert scoring. This method is too subjective. The entropy method uses the idea of information entropy to determine the weight of the index. Information entropy is used to measure the uncertainty of the index and can objectively reflect the effectiveness of the data.

The index information entropy of column $j$ in the decision matrix is as follows: 


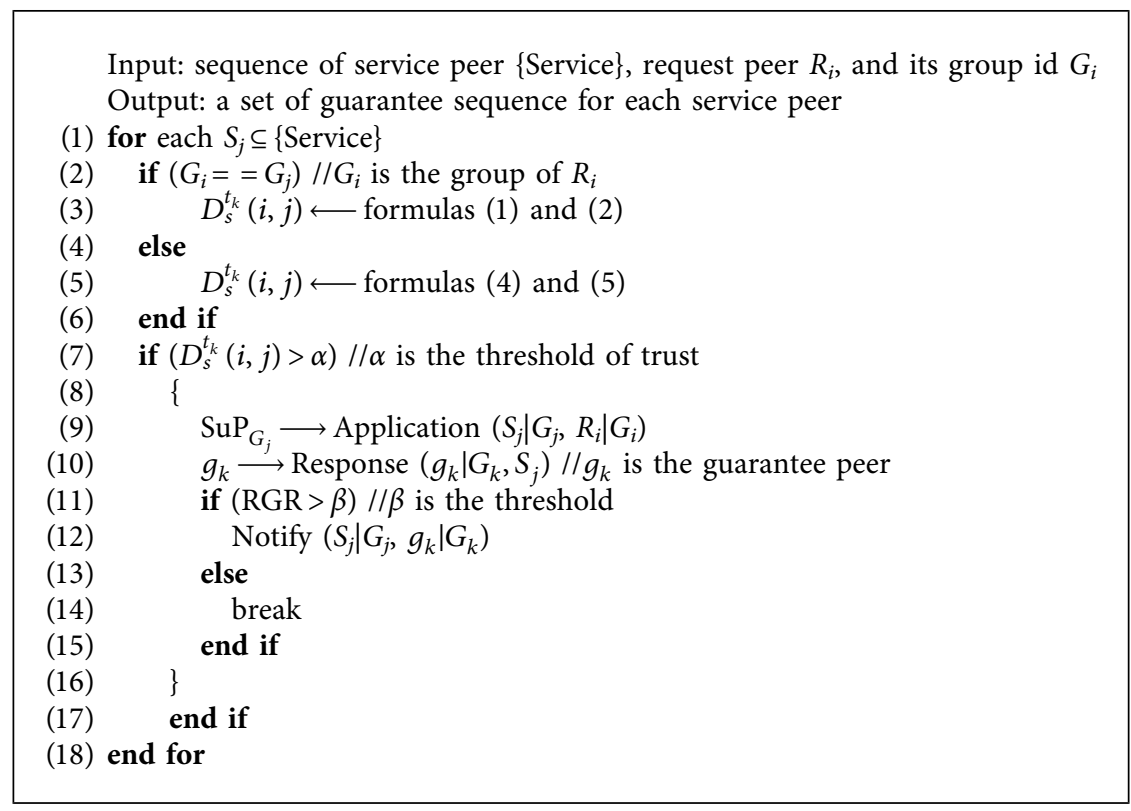

Algorithm 2: Selection of guarantee service peer.

TABLE 1: Evaluation indexes.

\begin{tabular}{lccccc}
\hline & $y_{1}$ & $y_{2}$ & $y_{3}$ & $y_{4}$ & $y_{5}$ \\
\hline$x_{1}$ & $y_{11}$ & $\ldots$ & $y_{13}$ & $\ldots$. & $y_{15}$ \\
$\ldots$ & $\ldots$ & $\ldots$ & $\ldots$ & $\ldots$ & $\ldots$ \\
$x_{i}$ & $y_{i 1}$ & $\ldots$ & $y_{i 3}$ & $\ldots$ & $y_{i 5}$ \\
$\ldots$ & $\ldots$ & $\ldots$ & $\ldots$ & $\ldots$ & $\ldots$ \\
$x_{m}$ & $y_{m 1}$ & $\ldots$ & $y_{m 3}$ & $\ldots$ & $y_{m 5}$ \\
\hline
\end{tabular}

$$
e_{j}=-k \sum d_{i j} \ln d_{i j}, \quad j=1, \ldots, 5
$$

where $k=1 / \ln m$. According to the basic principle of information entropy, the larger the amount of information that is carried by an index and the smaller the uncertainty, the smaller the entropy and the larger the weight. Then, the weight calculation formula of each evaluation index $w_{j}$ is as follows:

$$
w_{j}=\frac{1-e_{j}}{n-\sum_{j=1}^{5} e_{j}} .
$$

After determining the weight of each index, the initial decision matrix is weighted as follows:

$x_{i j}=w_{j} * Z_{i j}, \quad i=1, \ldots, m, j=1, \ldots, 5$

(4) Ideal service peer $x_{j}^{+}$and negative ideal service peer $x_{j}^{-}$is as follows:

$$
\begin{aligned}
& X_{j}^{+}= \begin{cases}\max _{i} x_{i j}, & j \text { is benefit attribute, } \\
\max _{i} x_{i j}, & j \text { is cost attribute, }\end{cases} \\
& X_{j}^{+}= \begin{cases}\max _{i} x_{i j}, & j \text { is cost attribute, } \\
\max _{i} x_{i j}, & j \text { is benefit attribute. }\end{cases}
\end{aligned}
$$

(5) The distance between service peer $X_{i}$ and the ideal service peer is calculated as follows:

$$
d_{i}^{+}=\sqrt{\sum_{j=1}^{n}\left(x_{i j}-x_{j}^{+}\right)^{2}} .
$$

The distance between service peer $X_{i}$ and the negative ideal service peer is calculated as follows:

$$
d_{i}^{-}=\sqrt{\sum_{j=1}^{n}\left(x_{i j}-x_{j}^{-}\right)^{2}} .
$$

(6) Determine proximity as follows: The proximity degree $C_{i}$ is the $i$ th index of the service peer, which is close to the ideal service peer and far away from the negative ideal peer. The specific calculation formula is given as follows:

$$
C_{i}=\frac{d_{i}^{-}}{d_{i}^{-}+d_{i}^{+}}, \quad(i=1,2, \ldots, m) .
$$

(7) Get the ideal trading peer sequence $\left\{S_{1}^{\prime}, S_{2}^{\prime}, \ldots, S_{m}^{\prime}\right\}$. 


\section{Calculation of Comprehensive Trust}

After obtaining the sequence of ideal transaction peers, the first $\left|\_m / 2_{-}\right|$service peers are selected to calculate their comprehensive trust. The comprehensive trust includes two aspects: one is global trust and the other is guarantee trust.

Reputation mortgage is required for each guaranteed peer $g_{j}$, and $\mathrm{RM}_{g i}$ of each guaranteed peer's required mortgage is calculated as follows:

$$
\mathrm{RM}_{g i}=\frac{\mathrm{RGR}_{g i} * e(1 / \text { Reward } * v)}{\sum_{j=1}^{V} \mathrm{RGR}_{g i}},
$$

where $\mathrm{RGR}_{g i}$ is the remaining guarantee reputation of guarantee peer $g_{j}$, Reward is the pay for this service, and $v$ is the number of guaranteed peers owned by the service peer.

$\mathrm{SuP}_{G_{i}}$ is the super peer of the group $G_{i}$, where $R_{i}$ is located. It needs to calculate the $\operatorname{Sim}\left(R_{i}, g_{i}\right)$ of the service similarity between the historical transaction records of the guaranteed peer $g_{i}$ and the request peer.

Let $g_{i}=\left\{a_{1}, a_{2}, \ldots, a_{n}\right\}$ and $R_{i}=\left\{b_{1}, b_{2}, \ldots, b_{n}\right\}$ be the historical service vectors of the guarantee peer $g_{i}$ and the request peer $R_{i}$ during the valid time, respectively.

Then, the similarity coefficient based on the cosine can be obtained as follows:

$$
\operatorname{Sim}\left(R_{i}, g_{i}\right)=\frac{\sum_{i=1}^{n} a_{i} \times b_{i}}{\sqrt{\sum_{i=1}^{n} a_{i}^{2} \times \sum_{i=1}^{n} b_{i}^{2}}} .
$$

The formula for calculating the guaranteed trust $\mathrm{GT}_{i}^{S_{j}}$ of service peer $S_{j}$ is as follows:

$$
\mathrm{GT}_{i}^{S_{j}}=\sum_{i=1}^{V} \sqrt[3]{\operatorname{Sim}\left(R_{i}, g_{i}\right) * \mathrm{RM}_{g_{i}} * d\left(t_{k}\right)}
$$

The comprehensive trust $\mathrm{CT}_{s_{j}}$ combines the direct trust with the guarantee trust. The specific formula is given by matrix as follows:

$$
\mathrm{CT}_{s_{j}}=\left(\begin{array}{ll}
w_{1} & w_{2}
\end{array}\right) *\left(\begin{array}{c}
D_{s}^{t_{k}}(i, j) \\
\mathrm{GT}_{i}^{S_{j}}
\end{array}\right),
$$

where $\omega_{1}+\omega_{2}=1$ and $\omega_{1}, \omega_{2} \subseteq[0,1]$, in which $\omega_{1}$ is the weight of the direct trust and $\omega_{2}$ is the weight of the guarantee trust. In social networks, request peers are more likely to choose peers that have multiple transactions with themselves to serve. Therefore, this paper takes the transaction impact function $f(k)$ as the weight assignment. The formula is as follows:

$$
f(k)=w_{j}= \begin{cases}1-\left(\frac{1}{2}\right)^{(k /(n-k))}, & n-k \neq 0, \\ 1, & n-k=0,\end{cases}
$$

where $k$ is the number of transactions between the request peer and the service peer and $f(k)$ is a function that dynamically adjusts with $k$. When $k=0$, it means that there is no direct historical transaction record between the two peers. At this time, the comprehensive trust should depend more on the guarantee trust.

\section{Reputation Update}

7.1. Feedback Verification. When the service is completed, the request peer will return a set of FMs (feedback messages). To discard dishonest feedback, the super peers of the group where the service peer and the guarantee peer are located need to verify the feedback FM.

In this paper, each feedback is regarded as a sample from a stationary normal process. The feedback of different request peers is independent and has no influence on each other. The historical feedback set $F=\left\{F_{1}, F_{2}, \ldots, F_{n T}\right\}$ of the request peer $R_{i}$ can be considered as a sample that follows a normal distribution. As a result, the average and variance of the peer $R_{i}$ are calculated in the nearest time interval $T$ to the feedback.

$$
\begin{aligned}
\bar{x} & =\frac{\sum_{i=1}^{n_{T}} F_{i}}{n_{T}}, \\
s^{2} & =\frac{\sum_{i=1}^{n_{T}}\left(F_{i}-\mu\right)^{2}}{n_{T}-1},
\end{aligned}
$$

where $n_{T}$ is the total amount of feedback information of $R_{i}$ in time interval $T$ and $F_{i}$ is the $i$ th feedback. The confidence level is 95\%. Only when FMs meet the following requirements can they be regarded as passing the verification:

$$
\mathrm{FM} \in\left[\bar{x} \pm \frac{s}{\sqrt{n_{T}}} t_{0.025}\left(n_{T}-1\right)\right] .
$$

7.2. Peer Reputation Update. When the service is completed and the FMs of the request peer are accepted, the reputation value of the service peer $S_{i}$ and its guarantee peer need to be updated. $\mathrm{FM} \subseteq[-1,1]$. If $\mathrm{FM} \subseteq[-1,0)$, the service is considered to be unsuccessful, and if $\operatorname{FM} \subseteq(0,1]$, the service is considered to be successful. To encourage service peers and guarantee peers to provide active services, this paper proposes a reward and punishment mechanism.

$$
\begin{aligned}
& \operatorname{reward}\left(g_{i}\right)=\frac{F_{g_{i}}^{\text {suc }}}{F_{g_{i}}^{\text {all }}} * \mathrm{RM}_{g_{i}}, \\
& \operatorname{reward}\left(S_{i}\right)=\frac{\sum_{j=1}^{V} F_{S_{i}}^{\text {succ }} / F_{S_{i}}^{\text {all }} * \operatorname{reward}\left(g_{j}\right)}{\sum_{j=1}^{V} \operatorname{reward}\left(g_{j}\right)},
\end{aligned}
$$

where $F_{g_{i}}^{\text {suc }}, F_{g_{i}}^{\text {all }}, F_{S_{i}}^{\text {succ }}$, and $F_{S_{i}}^{\text {all }}$ are, respectively, the numbers of successful transactions and the total numbers of transactions of peer $g_{i}$ or $S_{i}$ within the time interval $T$ nearest to the feedback.

The reputation of the guarantee peer is updated as follows:

$$
R_{g_{i}}^{\text {new }}=R_{g_{i}}^{\text {pre }}+\mathrm{FM} * \frac{1}{1+e^{- \text {reward }\left(g_{i}\right)}},
$$


where $R_{g_{i}}^{\text {new }}$ is the current reputation of the guarantee peer $g_{i}$ and $R_{g_{i}}^{\text {new }}$ is the updated reputation of the guarantee peer $g_{i}$.

The reputation of service peer $S_{i}$ is updated as follows:

$$
R_{S_{i}}^{\text {new }}=R_{S_{i}}^{\text {pre }}+\mathrm{FM} * \frac{1}{1+e^{-\operatorname{reward}\left(S_{i}\right)}},
$$

where $R_{S_{i}}^{\text {new }}$ is the current reputation of the service peer $S_{i}$ and $R_{S_{i}}^{\text {new }}$ is the updated reputation of the service peer $S_{i}$.

\section{Experimental Results and Analysis}

The simulation experiment mainly verifies whether the GTBidding algorithm selects the ideal trading peer in the groupbased P2P network environment. This article divides peer sets into the following three categories based on their behavior:

(1) Real Peer (RP). The real peer is true whether it is providing services, guarantees, or feedback

(2) Unreliable Peer (URP). Such peers provide true guarantees and feedback, but there is still a low probability of the unintentional provision of false services

(3) Malicious Peer (MP). This type of peer is specifically divided into the following subclasses:

(I) Complete Malicious Peer (CMP). This type of peer maliciously provides services, guarantees, and gives malicious feedback

(II) Defame Peer (DP). This type of peer provides real services and guarantees, but when used as a request peer, it will give poor feedback after the transaction is ended

(III) Strategic Malicious Peer (SMP). Such a peer actively provides real service when the reputation value is low and provides a malicious service when the reputation value is high

(IV) Oscillating Malicious Peer (OMP). This peer provides the irregular false services or malicious information in the early stage of the transaction.

Because the classification of malicious peers is very complex, different types of malicious services can be characterized as a new subset of malicious peers. This paper conducts simulation experiments and considers only the above three subsets.

8.1. Experimental Setting. This article uses PlanetSim 3.0 to simulate a P2P network. The simulated hardware environment is an Intel(R) Core(TM) i5-7200U $2.50 \mathrm{GHz}$ CPU. The simulation experiment environment is as follows.

We set the number of network peers to 1000, where the number of groups is 20 and the number of neighboring groups in each group is 3 5. Each peer can become a service peer, a request peer, or a guarantee peer as long as it meets the requirements of the GT-Bidding model. The service category is set to 1000 , thereby ensuring that each peer can provide at least one type of service. The number of simulations in this experiment is 10 times, the simulation period is 50 iterations, and the average value is taken as the experimental result. At least one interaction record is recorded in each simulation cycle, and the SAM is issued by the request peer. Each service peer needs at least one guarantee peer to perform the reputation mortgage. After the transaction ends, the request peer sends feedback information and updates the peer reputation according to the feedback. The initial trust value of $\alpha$ is set to 0.5 . This indicates that this peer is half-trusted and half-untrusted. $\beta$ is the trust threshold of guarantee node, which is set to 0.7. Other parameter settings are shown in Table 2.

8.2. Tests of Resisting Attacks. In Experiment 1, the GTBidding algorithm is compared with the PSRTrust model [9], the SGTM model [10], and the SecureTrust model [14] to verify whether the computational complexity of the proposed trust algorithm is lower. The experimental results are shown in Figure 3. This experiment mainly records the time that is spent by the three models after 50 simulation cycles. Both the SGTM and PSRTrust models are based on the recommendation-based trust model. The reputation value of the peer is calculated by collecting the evaluation of the target peer on the whole network. Although the behavioral attribute of the target peer can be grasped to some extent, the computational complexity is high. Historical trust and deviation reliability are used in the SecuredTrust model to solve the problem of malicious nodes alternately attacking the network. And the dynamic allocation of agent work is also realized. Therefore, it has the highest computational complexity. The GT-Bidding model adopts the form of the guarantee instead of the recommendation, does not need to collect the evaluations of the target peer in the whole network, and proposes the entropy TOPSIS selection method to filter and sort the service peers, which greatly reduces the computational complexity.

In Experiment 2, by changing the OMP ratio from $0 \%$ to $50 \%$, the other peers are set to RPs and URPs. The GTBidding with information entropy is compared with the SGTM model without information entropy, and the transaction success rate is used as the evaluation index. It can be seen from Figure 4 that the successful transaction rate of the SGTM model is significantly lower than that of the GTBidding model. Because GT-Bidding introduces the information entropy calculation weight, reduces the impact of objectivity, and makes the classification more accurate, it has a higher successful transaction rate than the SGTM model.

In Experiment 3, by changing the CMP ratio from 0 to $50 \%$, the other peers were set to RPs and URPs. The GTBidding is compared with the PSRTrust, SGTM model, and SecureTrust model, and the successful transaction rate is used as the evaluation index. It can be observed from Figure 5 that when the CMP ratio is $10 \%$, the successful transaction rates of the four models are not much different. When the CMP ratio is greater than $10 \%$, the successful transaction rate of the SGTM model shows a significant downward trend. Increasingly, public transaction records are decreasing and successful transaction rates are decreasing. 
TABLE 2: Parameter settings.

\begin{tabular}{lc}
\hline Parameter & Value \\
\hline$\alpha$ & 0.5 \\
$\beta$ & 0.7 \\
RP : URP : MP & {$[35 \%, 25 \%, 40 \%]$} \\
CMP :DP :SMP & {$[50 \%, 25 \%, 25 \%]$} \\
\hline
\end{tabular}

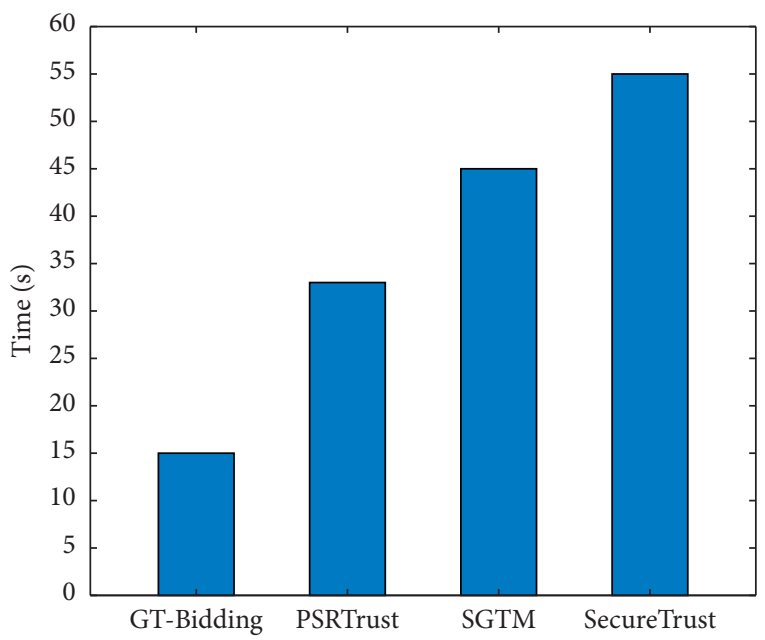

Figure 3: Comparison of the computational complexity of four types of models.

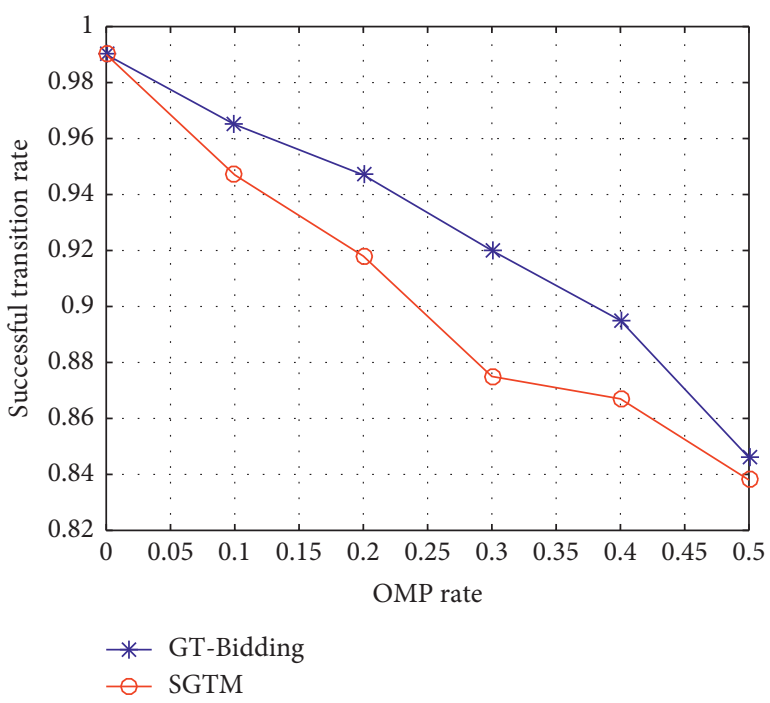

FIGURE 4: Comparison of the successful rate of GT-bidding and SGTM.

When the CMP ratio is between $10 \%$ and $30 \%$, the successful transaction rates of the PSRTrust and GT-Bidding models are similar and slightly better than those of the GTBidding model. This is because the PSRTrust model will prefer similarity and Markov randomization. The combination of walking strategies and CMP peers is more accurate, but when the proportion of CMP peers is $40 \%-50 \%$, similar random walk strategies cannot identify CMP peers in real

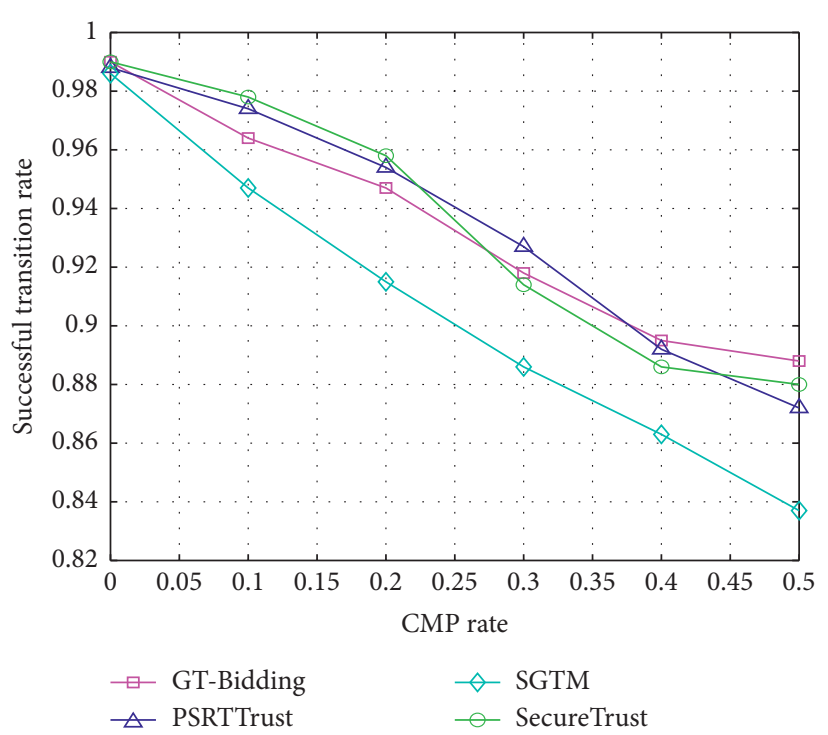

Figure 5: Successful transaction rates at different CMP ratios.

time. The GT-Bidding model evaluates the service peers before the transaction begins, greatly reducing the possibility of CMP peers providing services.

In Experiment 4, the DP ratio is gradually increased from $0 \%$ to $50 \%$, and the remaining peers are equally divided between RPs and URPs. DPs provide real services and guarantees but provide derogatory feedback. A DP has a high probability of forming a collusive attack, which means that the DP's reputation value may increase as the DP ratio increases.

As shown in Figure 6, when the DP ratio is between 10\% and $50 \%$, the successful transaction rate of the SGTM sharply drops. This is because the SGTM does not filter the feedback of services. Therefore, the larger the DP ratio is, the smaller the successful transaction rate is. The PSRTrust model identifies the conspiring group based on the activity similarity between the peers. Both PSRTrust and SecureTrust model identify collusion groups and filter out malicious feedback based on the activity similarity between nodes. The difference is that SecureTrust introduces a penalty factor in calculating feedback similarity, which makes its successful transaction rate higher than PSRTrust. However, these two models lack effective measures to resist collusion between recommending peers. The GT-Bidding model identifies the defamation peers based on historical feedback; therefore, the successful transaction rates of both are higher, but the PSRTrust model assesses the peers after the transaction. Due to collusion detection, at this time, the trust value of the RP peer will decrease. Meanwhile, GT-Bidding gives a specific 95\% confidence interval and only accepts feedback in the confidence interval, which can make the GT-Bidding calculation model more accurate. Discarding false feedback does not affect the trust value of the RP peer.

In Experiment 5, the SMP ratio is set from $0 \%$ to $50 \%$, and the remaining peers are RPs and URPs. The GT-Bidding algorithm is still compared with the PSRTrust, SGTM, and SecureTrust, with respect to the successful transaction rate. As seen from Figure 7, although the SGTM takes into 


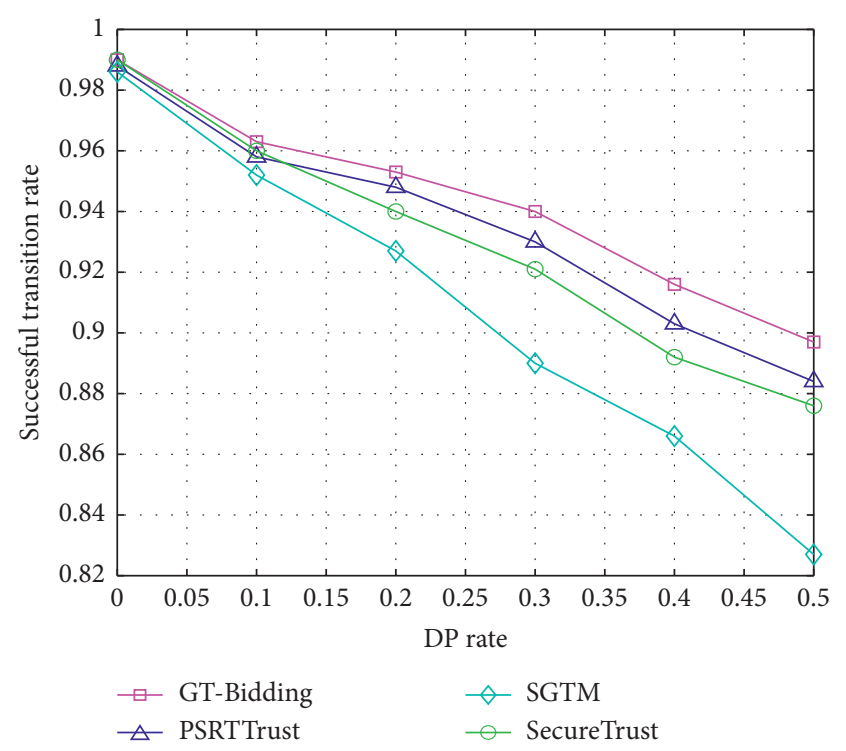

FIGURE 6: Successful transaction rate at different DP ratios.

account the time impact factor and risk factor, to a large extent, the global trust of the peer depends on past historical transaction records, and the request peer is easily deceived by SMPs, resulting in a significantly lower success rate. When the proportion of SMPs in the network is less than $20 \%$, the successful transaction rates of GT-Bidding and PSRTrust model are not much different. However, when the proportion of SMP peers is larger, the GT-Bidding model requires the trust degree and guarantee reputation of service peers to exceed the thresholds that must be met to participate in the bidding, which means that SMP peers need to provide a certain amount of real services before they can attack the network. If malicious services are provided, their reputation will be lower than the threshold.

When the proportion of SMP reaches $50 \%$, the successful transaction rate of SecureTrust is close to $90 \%$. Because it considers the credibility of feedback, historical trust, and deviation reliability, it can also effectively prevent malicious counterparts from strategically oscillating. However, SecureTrust is weak in resisting malicious feedback. The GTBidding model can provide services when the peers' accumulated reputation reaches a certain threshold. In other words, SMP must first pay a high cost before it can act maliciously. In turn, its malicious behavior will reduce its reputation below the threshold. Therefore, the GT-Bidding mode has a strong ability to resist strategic attacks.

In Experiment 6, the parameters of the simulation experiment are set as follows. The ratio of RP:URP:CMP : SMP : DP $=3: 2: 1: 3: 1$. Experiment 5 simulates the collusion of the service peer and the guarantee peer, as shown in Figure 8. It is found that with the continuous increase in the number of simulation cycles, the trust value of the CMP peer sharply drops and the trust values of the RP and URP peers steadily rise. The DP and SMP peers are stable around the 20th cycle, and the trust value almost no longer rises. Although this situation provides a possible service opportunity for a malicious service peer, the malicious service peer must

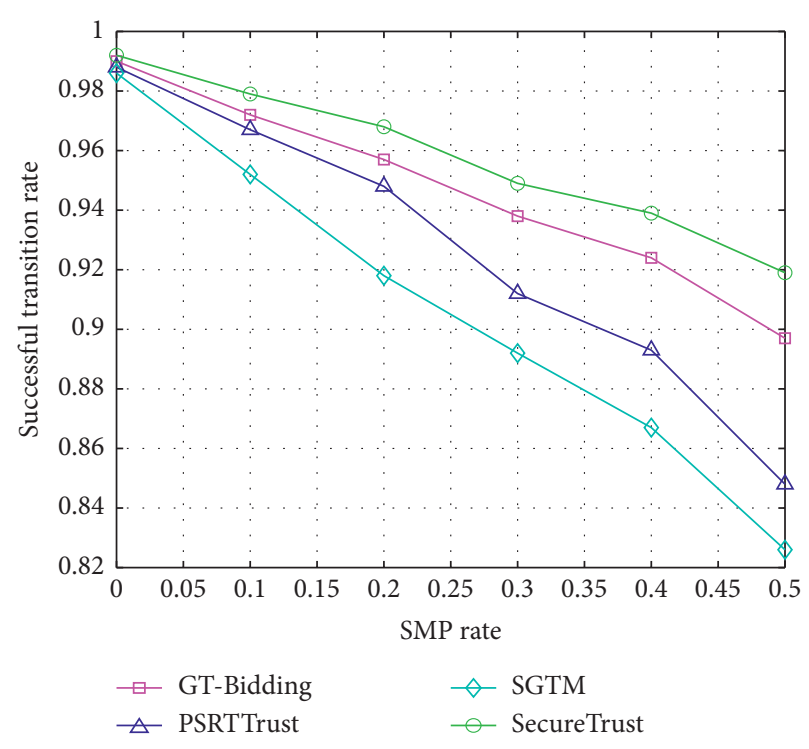

Figure 7: Successful transaction rates at different SMP scales.

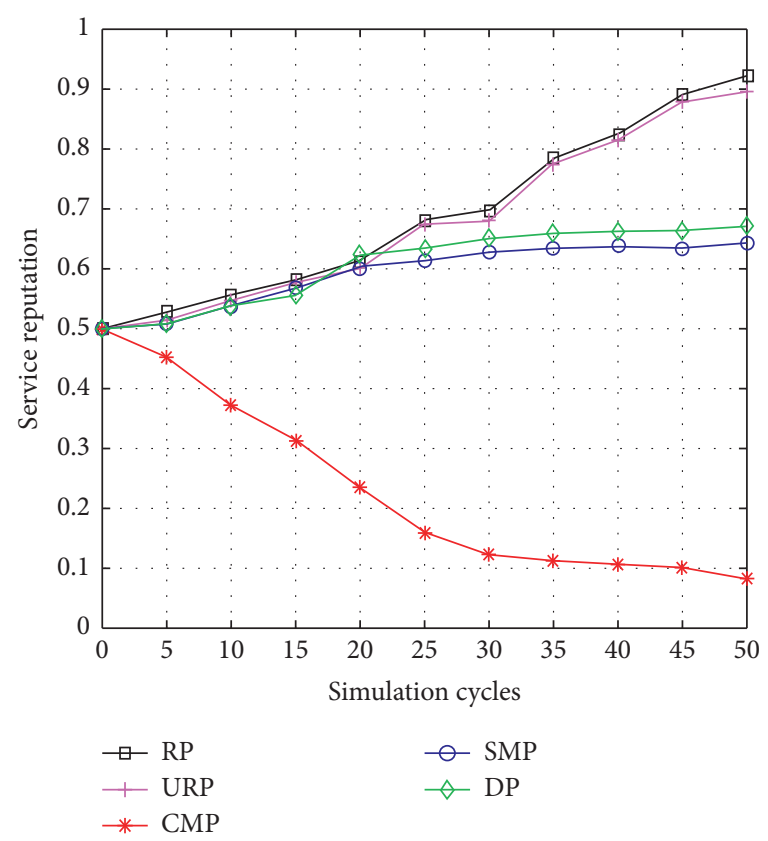

Figure 8: Changes in the trust values of various peers under a conspiracy attack of the service peer and the guarantee peer.

first pass the trust calculation to qualify as a possible complicity guarantee peer, which means that the malicious service peer must first accumulate a certain real service. Furthermore, the normal request peer gives real feedback after ending the transaction, which will reduce the trust value of the malicious service peer, and these peers will lose the opportunity to provide services and guarantees.

The data parameter setting of Experiment 7 is with the same as that in Experiment 6. Experiment 7 simulates a collusion attack between the service peer and the request peer. As seen from Figure 9, the trust value of the RP peer is continuously increased, that of the CMP peer is drastically 


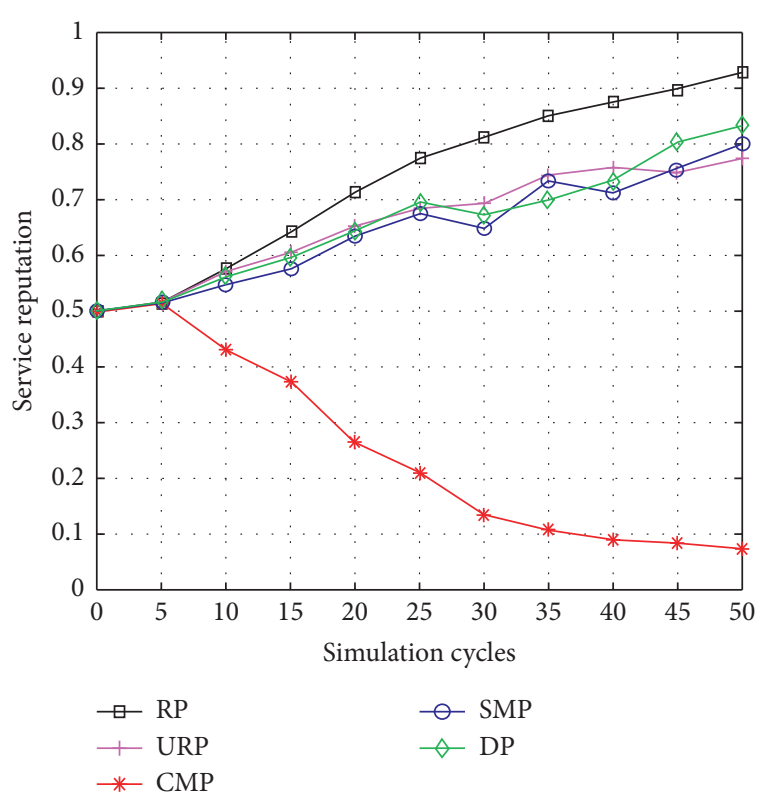

FIGURe 9: Analysis of resisting collusion attacks between service peer and request peer.

reduced, and the URP peer is trusted. The value is relatively stable when the number of simulation cycles is approximately 25. The trust values of the SMP peer and the DP peer begin to oscillate. This is because the request peer wants to establish a service relationship with the service peer, and the service gives false good feedback, thereby increasing the trust value of the companion peer in the GT-Bidding model. Due to bidding, the service peer needs to have at least one guarantee peer to guarantee it, and it is qualified to provide services through the layered filtering of the entropy TOPSIS selection method. Therefore, the possibility of a collusion service peer passing the screening is low.

\section{Conclusion and Future Work}

In this paper, based on the background of $\mathrm{P} 2 \mathrm{P}$ groups, a GTBidding trust model is proposed in which the competitive bidding mode is used to stimulate the enthusiasm of peers to provide real services, analogous to human society. First of all, the service peer needs to apply for a set of guarantee peers to mortgage the reputation of the service. Second, the entropy TOPSIS selection method is used to sort all the service peers participating in the bidding, and the ideal trading peer sequence is obtained. To reduce the computational complexity, the comprehensive trust degree is calculated only for the top peers in the sequence, and the transaction influence function is introduced into the comprehensive trust degree calculation to integrate the guarantee trust degree and the direct trust degree. Furthermore, to avoid false feedback, the feedback of the requesting peer is verified using the confidence interval based on the historical feedback set, and the reputation of the service peer and the guarantee peer is updated according to the effective feedback. Finally, the simulation results show that GT-Bidding is effective in improving the successful trading rate, suppressing malicious attacks and so on. However, with the addition of information entropy into the model, the success rate increases but the computational complexity also increases. And there is still a lack of discussion on the dynamic topology of mobile P2P nodes.

In our next study, we will further improve the GTBidding model, classify the guarantee peers, and discuss the dynamic topology of the peers in depth to improve the availability of the model in P2P networks.

\section{Data Availability}

The data used to support the findings of this study are included within the article.

\section{Conflicts of Interest}

The authors declare that they have no conflicts of interest.

\section{Acknowledgments}

This work was supported in part by the National Natural Science Foundation of China under Grant nos. 61572260, 61872196, and 61872194, in part by the Scientific \& Technological Support Project of Jiangsu Province under Grant no. BE2017166, and in part by the Jiangsu Government Scholarship for Overseas Studies.

\section{References}

[1] T. Wang, Y. X. Mei, X. X. Liu et al., "Edge-based auditing method for data security in resource-constrained internet of things," Journal of Systems Architecture, vol. 114, no. 5, Article ID 101971, 2020.

[2] X. F. Meng, "speedTrust: a super peer-guaranteed trust model in hybrid P2P networks," The Journal of Supercomputing, vol. 74, no. 6, pp. 2553-2580, 2018.

[3] J. H. Cho and I. R. Chen, "PROVEST: provenance-based trust model for delay tolerant networks," IEEE Transactions on Dependable and Secure Computing, vol. 15, no. 99, pp. 151$165,2018$.

[4] T. Wang, Y. Lu, J. Wang, H.-N. Dai, X. Zheng, and W. Jia, "EIHDP: edge-intelligent hierarchical dynamic pricing based on cloud-edge-client collaboration for IoT systems," IEEE Transactions on Computers, no. 99, p. 1, 2021.

[5] H. He, R. Li, X. Dong et al., "Secure, efficient and fine-grained data access control mechanism for P2P storage cloud," IEEE Transactions on Cloud Computing, vol. 2, no. 4, pp. 471-484, 2014.

[6] Y. Patel and N. Sethi, "Enhancing security in cloud computing using multilevel authentication," International Journal of Electrical Electronics \& Computer Science Engineering, vol. 1, no. 1, pp. 35-39, 2014.

[7] D. Kamvar and T. Schlosser, "EigenRep: reputation management in P2P networks," in Proceeding of the 12th ACM International World Wide Web Conference, pp. 123-134, Budapest, Hungary, May 2003.

[8] C.-Q. Tian, J.-H. Jiang, Z.-G. Hu, and F. Li, "A novel superpeer based trust model for peer-to-peer networks," Chinese Journal of Computers, vol. 33, no. 2, pp. 345-355, 2010.

[9] T. Shen, W. Tan, Y. Sun et al., "Identifying collusion attack based on preference similarity in mixed reputation recommendation model," Human Centered Computing, Springer 
International Publishing, vol. 74, pp. 2553-2580, Cham, Switzerland, 2016.

[10] X. Wu, "A stable group-based trust management scheme for mobile P2P networks," Journal of Computer, vol. 37, no. 10, pp. 2119-2127, 2014, in Chinese.

[11] G. Wang and J. Wu, "Multi-dimensional evidence-based trust management with multi-trusted paths," Future Generation Computer Systems, vol. 27, no. 5, pp. 529-538, 2011.

[12] B. Qureshi, G. Min, D. Kouvatsos et al., "A distributed reputation and trust management scheme for mobile peer-topeer networks," Computer Communications, vol. 35, no. 5, pp. 608-618, 2012.

[13] A. O. Samah, H. N. Kim, and A. E. Saddik, "A group trust metric for identifying people of trust in online social networks," Expert Systems with Applications, vol. 39, no. 18, pp. 13173-13181, 2012.

[14] A. Das and M. Islam, "SecuredTrust: a dynamic trust computation model for secured communication in multiagent systems," IEEE Transactions on Dependable and Secure Computing, vol. 9, no. 2, pp. 261-274, 2012.

[15] Y. Hu, D. Wang, H. Zhong et al., "SocialTrust: enabling longterm social cooperation in peer-to-peer Ss," Peer-to-Peer Networking and Applications, vol. 7, no. 4, pp. 525-538, 2014.

[16] X. F. Meng, "sureTrust: a super peer-aware trust model for 2level P2P networks," Peer-to-Peer Networking and Applications, vol. 11, no. 3, pp. 619-631, 2017.

[17] K. Bendiab, S. Shiaeles, and S. Boucherkha, "FCMDT: a novel fuzzy cognitive maps dynamic trust model for cloud federated identity management," Computers \& Security, vol. 86, no. 6, pp. 270-290, 2019.

[18] Y. C. Liu and Y. H. Liang, "Dynamic P2P trust modelbased on context factors," Journal on Communications, vol. 37, no. 8, pp. 34-45, 2016, in Chinese.

[19] M. Yurdakul and Y. Tansel, "Comparison of fuzzy and crisp versions of an AHP and TOPSIS model for nontraditional manufacturing process ranking decision," Journal of Advanced Manufacturing Systems, vol. 18, no. 2, pp. 167-192, 2019.

[20] O. A. Wahab, J. Bentahar, and H. Otrok, "Towards trustworthy multi-cloud services communities: a trust-based hedonic coalitional game," IEEE Transactions on Services Computing, vol. 11, no. 99, pp. 184-201, 2018.

[21] Z. C. Ma, L. Liu, and W. Z. Meng, "Towards multiple-mixattack detection via consensus-based trust management in IoT networks," Computers \& Security, vol. 96, Article ID 101898, 2020.

[22] W. Z. Meng, W. J. Li, and L. Q. Zhu, "Enhancing medical smartphone networks via blockchain-based trust management against insider attacks," IEEE Transactions on Engineering Management, vol. 67, no. 4, pp. 1377-1386, 2020.

[23] N. Narang and S. Kar, "A hybrid trust management framework for a multi-service social IoT network," Computer Communications, vol. 171, no. 11, pp. 61-79, 2021.

[24] Z. Yang, T. Yang, T. Fan et al., "Cloud service composition based on trust combination," Journal of Electronics, vol. 46, no. 3, pp. 614-620, 2018.

[25] P. Zhang, M. C. Zhou, and Y. Kong, "A double-blind anonymous evaluation-based trust model in cloud computing environments," IEEE Transactions on Systems, Man, and Cybernetics: Systems, vol. 51, pp. 1-12, 2019.

[26] W. J. Li, J. Cao, K. Y. Hu et al., "A trust-based agent learning model for service composition in mobile cloud computing environments," IEEE Access, vol. 7, pp. 2169-3536, 2019. 